\section{Hybridisation of ScDNA does not lead to overestimates of mRNA complexity}

THE contention of Kiper $^{1}$, that complexity estimates of mRNA populations measured by hybridisation with single copy DNA (scDNA) are routinely overestimates, is not supported by previously published data and additional data we provide here. Kiper argues that selective loss of single copy sequences (cryptic unique sequences) occurs when scDNA is isolated from randomly sheared DNA because they are adjacent to repetitive sequences. He contends that the loss is selective for sequences which do not code for messenger RNA, and hence scDNA is enriched in sequences which code for mRNA. Thus complexity measurements on mRNA based on scDNA hybridisation result in overestimates.

From data on the sea urchin genome ${ }^{2}$ it can be calculated that $\sim 19 \%$ of the total genomic DNA might be present as 'cryptic' scDNA when 450-nucleotide fragments are used in the preparation of scDNA. Assuming all of the cryptic scDNA to be non-code sequence, the resulting scDNA would be enriched for code sequences such that saturation hybridisation data might yield a complexity estimate that is too high by a factor of $\sim 1.3$, which is less than suggested by Kiper. A more important consideration is the likelihood that code sequences are extensively represented in cryptic scDNA. For example $80-100 \%$ of the mass of the mRNA in sea urchin embryos is complementary to scDNA sequences which are adjacent to interspersed repetitive sequences $^{3}$. If this is also true for complexity, and if the sequence organisation of sea urchin DNA is typical of eukaryotic genomes, then cryptic scDNA would contain code sequences and may even be somewhat enriched for these sequences. Whatever the case, a portion of the cryptic scDNA is expected to be code sequences. We have also found, using $S_{1}$ nuclease, that only about $10 \%$ of mouse DNA considered to be repetitive by hydroxyapatite (HAP) analysis of renaturation kinetics may be cryptic scDNA. Thus the extent of code sequence enrichment of scDNA suggested by Kiper seems unlikely.

Kiper's second point, that assay of DNA/RNA hybrids by HAP chromatography leads to overestimates due to the non-base-paired intervening sequence being present in DNA/RNA hybrids, is an important consideration in light of recent studies which suggest that several known structural genes are discontinuous in the genome ${ }^{4-6}$, and perhaps, as well, a large number of unidentified mRNA coding sequences ${ }^{7}$. However, values obtained by HAP and $S_{1}$ nuclease assays of hybridised scDNA are similar (Table 1). It is evident from this comparison that a negligible portion of the hybridised scDNA is not base paired, as has been previously reported $^{8}$. Despite these results we concur that $S_{1}$ nuclease method of assay is preferable to the use of HAP for reasons presented elsewhere?

From the aspects mentioned above Kiper suggests that the discrepancy between the scDNA and the cDNA methods of estimating the complexity of mRNA populations is largely due to gross overestimates generated by the scDNA method. From our results we find the discrepancy to be due more to the inaccuracy of the $C_{0} t_{2} \frac{1}{2}$ determinations for the most complex class of mRNA which is particularly imprecise if the cDNA is only partially hybridisable (that is, $\sim 75 \%$ or less). We prepared cDNA complementary to mouse brain poly $(\mathrm{A})^{+} \mathrm{mRNA}$ which was hybridisable to $91-92 \% \mathrm{~S}_{1}$ resistance. From hybridisation kinetics the complexity of the poly $(\mathrm{A})^{+} \mathrm{mRNA}$ was estimated to be $1.1 \times 10^{5}$ kilobases $^{10}$, a value which is similar to that obtained by the scDNA hybridisation method (Table 1). We conclude that the scDNA hybridisation method does not lead to gross overestimates of the complexity of RNA populations for reasons offered by Kiper.

\section{WILLIAM E. HAHN JEFFREY VAN NESS IAN H. MAXWELL}

\section{Department of Anatomy,}

University of Colorado,

School of Medicine,

Denver, Colorado 80262

1. Kiper, M. Nature 278, 279-280 (1979).

2. Graham, D. E., Neufeld, B. R., Davidson, E. H. \& Britten, R. J. Cell 1, 127-138 (1974).

3. Davidson, E. H., Hough, B. R., Klein, W. H. \& Britten, R. J. Cell 4, 217-238 (1975).

4. Dugaiczyk, A. et al. Nature 274, 328-333 (1978)

5. Matthyssens, G. \& Tonegawa, S. Nature 273, 763-765 (1978).

6. Tilghman, S. M., Curtis, P. J., Tiemeier, D. C., Leder, P. \& Weissman, C. Proc. natn. Acad. Sci. U.S.A. 75, 13091313 (1978).

7. Maxwell, I. H. \& Hahn, W. E. J. supramolec. Struct. Suppl. 3, 71 (1979).

8. Bantle, J. A. \& Hahn, W. E. Cell 8, 139-150 (1976).

9. Maxwell, I. H., Van Ness, J. \& Hahn, W. E. Nucleic Acid Res. 5, 2033-2038 (1978).

10. Hahn, W. E., Van Ness, J. \& Maxwell, I. H. Proc. natn. Acad. Sci. U.S.A. 75, 5544-5547 (1978).

HYBRIDISATION of labelled single copy DNA with an excess of RNA is an important procedure for estimating the number of genes expressed in cells ${ }^{1}$. Recently, Kiper ${ }^{2}$ asserted that gene number values in plants obtained by using this method are routinely significant overestimates. To support his premise, Kiper cited our measurements of the number of genes expressed in the tobacco leaf ${ }^{3}$. We contend that our original interpretation of the tobacco data ${ }^{3}$ is theoretically sound and that the re-interpretation by $\mathrm{Kiper}^{2}$ is based on specious arguments.

Kiper's first argument maintained that a large fraction of interspersed single copy DNA (scDNA) in plant genomes consisted of $\leqslant 300$-base pair, unexpressed sequences. During the kinetic isolation of scDNA, these short, interspersed, unique sequences would be selectively lost due to their close linkage with repetitive sequence elements. The effective result (as Kiper maintains), therefore, would be an enrichment for expressed sequences in the single copy preparations used for DNA/RNA hybridisation experiments. Most plant genomes investigated to date, however, do not possess very short interspersed single copy sequences ${ }^{4}$. For example, the vast majority of interspersed single copy sequences in tobacco ${ }^{5}$, soybean $^{6-8}$, cotton $^{9}$, corn (S. Hake and V. Walbot, unpublished), wheat $^{10}$, and mungbean $^{11}$ exceed 1,000 base pairs. Only in pea ${ }^{12}$ and perhaps rye ${ }^{13}$ does there seem to be a fraction of short scDNA. Direct electron microscopic observations have shown that the minimum number average lengths of interspersed single copy sequences in soybean ${ }^{7}$ and cotton ${ }^{9}$ are 1,300 and 2,900 base pairs respectively. Few short ( $<500$ base pairs) single copy sequences were visualised $(<5 \%)$. As the fragment length (200-300 bases) of scDNA used in gene expression studies is much less than that of the interspersed unique DNA, it is unlikely that a separate complexity class of unexpressed, 'cryptic', single copy sequences will be removed during scDNA isolation. The scDNA mass that is lost (due to short period interspersion effects) will simply be that which is at the termini of moderately long scDNA regions. Such regions have been demonstrated to code for mRNA ${ }^{14-16}$. We argue, therefore, that short plant scDNA preparations of the type used in RNAexcess/scDNA hybridisation experiments will not be enriched for expressed sequences or any other sequences as Kiper asserts.

Kiper's second argument was based on recent observations which have demonstrated that several animal genes are interrupted by non-coding sequences (intervening sequences) $)^{17}$. He contends, correctly, that if intervening sequences are present in plant genomes, they could potentially lead to an overestimate of gene number when DNA/RNA hybrids are assayed by binding to hydroxyapatite. However, as shown in Table 2 this is not the case, as similar gene number values were obtained with the scDNA method 